\title{
La collaboration entre praticiens et chercheurs
}

\author{
Aurélie Valtat, \\ Digital Strategist, \\ Commission Européenne \\ Sandrine Roginsky, Professeure, \\ Université catholique de Louvain \\ Sandrine.roginsky@uclouvain.be
}


Aurélie Valtat est responsable de la communication digitale au Conseil de l'Union européenne, l'une des trois principales institutions de l'Union européenne. Elle est membre et ancienne présidente de l'IABC (International Association of Business Communicators). Titulaire d'un DEA en Sciences politiques, elle a essentiellement travaillé dans les organisations internationales, où elle s'est formée à la communication numérique.

1) Les travaux des chercheurs sont-ils utiles et accessibles au praticien de la communication que vous êtes?

Dans mes différents métiers, j'ai toujours fait appel aux travaux scientifiques : j'ai beaucoup lu sur les théories de la communication par exemple. J'utilise beaucoup les travaux de recherche, mais c'est vrai que, pour ce qui concerne spécifiquement la communication numérique, le contenu sur lequel je travaille vient essentiellement des Etats-Unis, du Canada, de Grande-Bretagne. Quand je cherche des informations, je cherche en anglais.

Beaucoup d'articles ne sont pas vendus comme de la recherche mais se nourrissent de recherche. Ce sont des praticiens qui lisent beaucoup de recherches, et traduisent cette recherche: ils répondent à des questions concrètes des praticiens en se nourrissant de cette recherche. Ces travaux ne m'arrivent pas directement. J'ai par conséquent l'impression que je suis toujours confrontée à la recherche par des intermédiaires. Ainsi, les travaux des chercheurs m'arrivent également par l'intermédiaire d'associations de professionnels ou de fédérations, notamment par le biais de leurs magazines, qui sont certes à destination des professionnels mais qui invitent des chercheurs. A titre d'exemple, la publication de l'Association européenne des directeurs de communication (EACD), «Communication Director» est intéressante: on y trouve une rubrique qui consiste en un dialogue entre un chercheur et un praticien sur un thème spécifique. Les chercheurs interviewés le sont sur la base d'un travail concret qu'ils ont conduit. Autrement dit, un cas spécifique est analysé par le praticien et le chercheur: ce dernier amène alors un éclairage académique. L'approche est par conséquent davantage de l'ordre de l'utilitaire. 
240 Le communicateur bousculé par le numérique

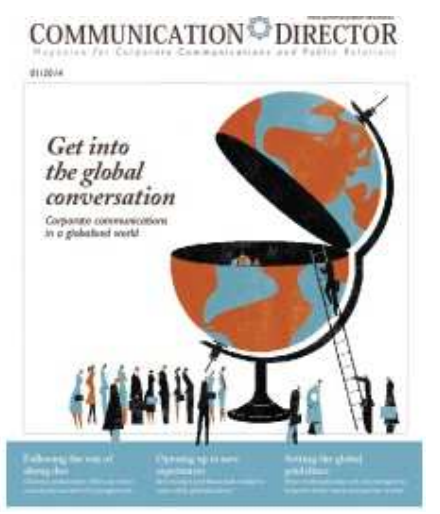

FIGURE 17

Exemple de couverture de la revue professionnelle « Communication

Director »

J'utilise aussi les ressources de l'association IABC, notamment en filtrant mes recherches via la catégorie «research and measurement» (http://www.iabc.com/resources/).

Je consulte aussi de temps en temps des revues académiques, comme Communication \& Organisation. A titre d'exemple, je me suis récemment appuyée sur un article scientifique publié par «The Public Relations Journal» pour documenter la manière dont les infographies peuvent être lues et comprises par les usagers et me permettre ainsi de réfléchir à l'impact des infographies que nous produisons dans le cadre de notre communication digitale. Cette revue a justement comme objectif de faciliter le transfert de connaissance entre la communauté académique et la communauté professionnelle. Pour que j'y ai accès, les articles scientifiques doivent être en open access. Pour ce qui est de Public Relations Review, tout n'est pas en open access : l'article phare l'est et quelques autres. Ca ne m'est jamais arrivé de payer pour un article scientifique, mais c'est d'abord parce que ce n'est pas pratique. S'il y avait un bouton "payer avec PayPal un article à deux euros ", ça serait plus facile. Cela dit, le prix est une limite. Pour un praticien ce n'est pas possible d'aller au-delà de quelques euros. Il est prêt à payer trois à quatre euros pour son usage personnel. Au-delà, ça doit passer par l'entreprise et là il doit justifier. Mais je ne suis pas sûre qu'il soit possible de justifier l'achat d'un article académique s'il n'y a pas un objectif opérationnel derrière. Si l'objectif est davantage de l'ordre de la connaissance personnelle, si l'organisation ne voit pas 
que ça a un impact au niveau du bottom line, je ne suis pas sûre que l'entreprise suive. Je crois que dans ce cas elle préférera envoyer le praticien en formation.

Par ailleurs, si ces articles sont enrichissants et utiles, il me manque parfois des éléments qui vont au-delà cette première approche d'analyse d'un cas concret. J'aimerais avoir aussi accès à des analyses plus approfondies, par exemple sur des aspects plus sociétaux. Mais je ne sais pas où aller les chercher. L'aspect éthique de la communication, notamment, est je pense un objet d'étude sur lequel les chercheurs travaillent: les praticiens à l'inverse ne l'abordent pas, ou très peu, au quotidien. Or, il s'agit d'une question importante. Y réfléchir en amont peut nous permettre d'anticiper les débats de société et ainsi préparer les praticiens de la communication. Ceux-ci peuvent se trouver confrontés à des questions éthiques, comme par exemple l'impact d'une communication sur des usagers et sur leur manière de « consommer » les médias.

2) Les chercheurs et les praticiens de la communication savent-ils communiquer et collaborer?

Il est plus courant de voir des praticiens venir à l'université, pour y donner des cours, que des chercheurs venir «pratiquer» en entreprise. Les modèles de collaboration sont peut-être à améliorer pour que les praticiens enrichissent le travail des chercheurs et pas seulement l'enseignement universitaire.

Dans l'autre sens, selon les axes de recherche des chercheurs, il peut y avoir une vraie valeur ajoutée pour les praticiens : le résultat des recherches scientifiques peut leur apporter des éléments intéressants, notamment quand ces recherches portent sur leur organisation et/ou leurs pratiques. Mais il faut alors réfléchir à la forme que peut prendre la transmission du chercheur au praticien: peut-être que des petits déjeuners thématiques pourraient être une manière de faire.

L'apport du chercheur vient notamment de son positionnement : il n'est pas dans l'organisation, il interroge, il regarde. Être à l'extérieur de l'organisation est un atout. Certaines choses peuvent être plus aisément dites par un chercheur extérieur que par des personnes qui, à l'intérieur de l'organisation, le répètent à corps et à cris depuis des années. Le chercheur pourra être davantage écouté et son discours pris en compte. Il peut jouer d'un statut, souvent prestigieux, et d'un sens de l'intérêt public. Le chercheur a normalement - comme le fonctionnaire - l'intérêt général à cœur. Il ne travaille pas pour une entreprise dont il cherche à faire valoir les intérêts.

Pour toutes ces raisons, la conduite d'audits par des chercheurs me semble être une très bonne idée : les chercheurs peuvent ainsi enrichir leurs travaux en collectant des données originales via les audits tout étant confrontés à la vie de l'entreprise. 
3) Est-ce que le positionnement du chercheur, extérieur à l'organisation, lui permet davantage de confirmer et valider ce que l'organisation sait déjà ou plutôt d'amener un regard différent et de proposer une approche qui n'est pas celle développée par ses praticiens?

Le premier cas est déjà celui d'un consultant. On fait appel à un consultant extérieur pour faire dire par quelqu'un d'extérieur des choses qu'on sait déjà en interne. Donc je ne pense pas que le chercheur apporte plus par rapport à ça.

Je pense que ça dépend de l'objectif du chercheur. Il y a des chercheurs qui vont utiliser leur passage dans l'organisation comme une possibilité d'observer quelque chose qui va nourrir leur recherche, et en même temps proposer des services d'expertise. Dans ce cas là, ils ne peuvent pas vraiment amener un regard académique de recherche dans leur travail d'expertise mais par contre, ils vont se nourrir de ce qu'ils ont appris pour leur propre recherche. Dans d'autres cas il peut y avoir effectivement un besoin d'approfondir certaines approches ou certaines techniques, mais là je ne connais pas assez l'implication des chercheurs pour savoir si les besoins des chercheurs et ceux de l'entreprise peuvent se rencontrer. Est-ce qu'une entreprise est amenée à se poser elle-même des questions profondes sur la manière dont elle opère en terme de communication, marketing ? Je ne sais pas, je ne connais pas de cas. Ce qui m'intéresserait si j'étais une entreprise serait que le chercheur fournisse certes son travail d'expertise, et que ce qu'il a appris lors de son passage dans l'organisation, il l'utilise ensuite pour un travail de recherche, mais de manière indépendante. Son analyse pourra être alors très critique, et peut-être qu'il ne sera pas ré-engagé par l'entreprise, mais au moins il aura donné un regard neutre, qui pourra toujours servir à ladite entreprise. Mais je vois mal comment un chercheur peut être indépendant si en même temps il est payé. Certes, il peut aussi ne pas être payé. Mais dans ce cas on n'est plus dans un « donnant-donnant ». Il peut avoir des financements qui viennent d'ailleurs, bien sûr, mais à ce moment là je questionnerais l'usage des fonds : est-ce que l'objectif de fonds publics est de faire en sorte qu'une entreprise gagne plus d'argent?

4) Comment les chercheurs peuvent-ils s'adresser aux praticiens? Quels supports sont les mieux adaptés?

J'aimerais bien qu'il y ait des blogs de référence, un peu sur le modèle du blog de Mickael Malherbe sur la communication européenne (http://www.lacomeuropeenne.fr/). Celui-ci n'est pas chercheur mais tente d'analyser la communication européenne.

Je vois ici deux formules: plusieurs chercheurs se mettent ensemble pour communiquer autour des thèmes de la communication; un chercheur particulier a 
une visibilité certaine dans son domaine. Mais j'aime bien cette idée de pouvoir régulièrement me nourrir de réflexions scientifiques, mais dans un langage qui reste abordable. Rendre le langage abordable, c'est le défi qui se pose aux chercheurs. Si on veut toucher un plus grand public de praticiens, il est nécessaire de transformer un peu le langage. Ce qui fait la spécificité et le sérieux du travail de recherche doit bien sûr rester, je pense ici aux références notamment, mais il faut peut-être utiliser plus des résumés, des executives summary d'une page ou demi-page, pour présenter l'essentiel - même si le fond de la recherche peut être présenté de manière plus substantielle, à l'exemple d'un article universitaire.

Certains praticiens sont intéressés par des éléments qui ne sont pas uniquement opérationnels, mais qui peuvent par exemple davantage porter sur la manière dont on travaille. Le côté méta autour d'une pratique est important parce qu'il nous permet, à nous praticiens, de réfléchir à nos assumptions, aux bases à partir desquelles on définit nos pratiques. Donc c'est intéressant, cela permet de se remettre en question et de s'enrichir. Mais bien sûr il faut au départ être un praticien intéressé par ce type de réflexions.

J'ai l'impression qu'il y a quand même un fossé - je n'arrive pas à trouver un mot plus neutre - entre les praticiens et les chercheurs. Nous sommes souvent, voire toujours, amenés à travailler avec des contractants externes (pour livrer des produits, pour réfléchir, pour faire des audits, etc.), qui ne sont jamais des chercheurs. Par contre, les agences avec lesquelles nous travaillons se nourrissent de recherches et peuvent même faire appel à des chercheurs; ce qui, selon moi, ajoute de la valeur à l'agence. Par exemple, l'agence avec laquelle on travaille pour le transmonitoring digital incorpore des résultats de recherche dans leurs outils. Autrement dit les outils qu'ils développent pour faire de la veille sont des outils très scientifiques. Je trouve cette approche rassurante. Je pense que les agences ont tout intérêt à travailler de manière plus étroite avec les chercheurs, pour développer des outils, des méthodologies qui ensuite deviennent des arguments de vente, mais aussi pour accompagner le travail en entreprise. Elles peuvent ainsi confronter les idées des praticiens avec celles des chercheurs. Je pense que c'est un argument de vente pour les agences, et c'est un moyen pour les chercheurs de compléter leur travail en se confrontant à des cas concrets.

Bien sûr rien n'empêche les académiques de créer des agences dont la fonction est spécifiquement de fournir des chercheurs. Par contre, cela me semble plus difficile de le faire seul, de manière isolée. Si les chercheurs ensemble créent des réseaux et promeuvent ce type de travail, ils auront plus de poids. Je vois difficilement comment réduire le fossé si le chercheur est seul et ne peut pas se prévaloir du réseau : le nombre est ici important pour construire la légitimité.

Un autre défi qui se pose aux chercheurs est la temporalité : les recherches peuvent se dérouler sur un temps long, parfois déconnecté de l'actualité. Mais les praticiens ont également besoin d'un éclairage plus distancié et réflexif de l'actualité. Il n'est 
pas rare qu'un chercheur soit interrogé à titre d'expert dans la presse généraliste : le chercheur travaille depuis longtemps sur un sujet mais doit réagir "à chaud» sur une actualité. Les blogs devraient également permettre aux chercheurs de commenter et éclairer l'actualité.

5) Est-ce qu'il serait utile aux praticiens que les chercheurs traduisent leurs travaux sous forme de manuels expliquant leurs méthodes et leurs outils?

Je crois que ça serait pas mal, mais ce qui serait encore plus utile serait des conférences ou des ateliers. Avec la lecture il y a toujours un degré d'interprétation et tout un contexte autour. Le plus intéressant serait d'avoir un contact direct avec les chercheurs. L'idée qu'on a souvent des chercheurs, c'est qu'ils sont détachés de la matière et détachés de la pratique au quotidien. J'ai peur que le format livre continue à alimenter ce cliché. Je pense que s'il y a une interaction physique, avec des échanges de bonnes pratiques, et avec une confrontation entre la vision de chercheurs, de praticiens qui ont mis en œuvre leurs idées et d'autres qui ont testé mais pour qui ça n'a pas fonctionné, il y a une vraie valeur ajoutée en terme d'échange. Les livres c'est bien aussi, mais je ne suis pas sûre que ça véhicule vraiment cette idée que «c'est pratique».

6) Comment les formations universitaires en communication peuvent-elles répondre aux besoins et demandes des praticiens?

Il y a, selon moi, deux axes principaux d'enseignement.

Il y a d'une part les méthodes et les techniques. Cet axe, je pense, s'appréhende mieux sur le terrain. Mais on peut apprendre du terrain si on l'a étudié en amont par des analyses d'études de cas et par des stages. C'est la partie plus pratique, elle est selon moi nécessaire dans l'enseignement. Mais bien sûr elle ne doit pas être approchée seulement par la constitution d'une «boite à outils ». Chaque outil doit être analysé, interrogé, réfléchi. Par exemple, on ne peut pas se limiter à expliquer qu'un communiqué de presse doit avoir un titre, un chapeau, tant de caractères, etc. Il faut surtout expliquer le pourquoi du comment: comment les gens lisent un communiqué de presse, quels sont les mécanismes de compréhension d'un document, etc. Pour ce faire, les académiques doivent s'appuyer sur les recherches scientifiques existantes. La professionnalisation du métier de communicant passe par là. Le praticien doit apprendre à utiliser des études pour faire le choix des outils pour produire des messages et de la communication.

Il y a ensuite un axe plus réflexif, où l'on trouve notamment la question des impacts de la communication: impact éthique, impact juridique, etc. Doit notamment y être abordée la question de la protection de la vie privée : jusqu'où peut-on aller pour étudier des contenus multimédias? 
Je pense que les méthodes et les techniques évoluent tellement vite, notamment à travers l'usage des réseaux sociaux et du web, qu'il est difficile d'enseigner cette partie à l'université, si ce n'est par la mise en situation et le stage. Personnellement, je n'ai pas suivi de formation universitaire en communication mais en sciences politiques. J'ai appris les techniques de communication et me suis familiarisée aux outils dans le cadre professionnel, donc le fait de ne pas avoir suivi de formation universitaire en communication ne m'a pas pénalisée. Au niveau exécution, je ne pense pas que retourner à l'université m'amènerait plus que ce que je peux acquérir à travers l'expérience professionnelle.

Ce qui parfois peut me manquer est davantage d'ordre argumentatif: les arguments pour justifier telle ou telle direction stratégique dans ma communication. Les choix que je fais peuvent parfois être davantage de l'ordre de l'intuitif : j'ai testé tel outil dans telle situation, ça a donné tel résultat, j'oriente ou ré-oriente en fonction. Je m'appuie sur mon expérience et sur les signaux que je capte de l'environnement immédiat, je les traduis de manière un peu intuitive mais je n'ai pas d'argument scientifique. Je pense que cela me serait utile; utile car tous les dirigeants ne sont pas sensibles aux mêmes arguments. Certains dirigeants vont être sensibles à l'expérience, d'autres aux projections chiffrées, d'autres aux arguments scientifiques du type « quand les gens lisent le communiqué de presse, $90 \%$ s'arrêtent à la fin du chapeau ». Plus tu as de cordes à ton arc, plus tu as de chance de mener à bien des propositions stratégiques.

Pour moi, la recherche, en tout cas dans le web digital, est un pré-requis de prise de décision. On essaie de mettre en place des méthodologies LEAN, à savoir on a une hypothèse, on la teste, on mesure et ensuite on décide si on continue dans cette direction ou si on pivote et on va vers une autre approche. Plutôt que de partir sur une idée et construire toute la stratégie et le produit à partir de celle-ci, on commence avec ce qu'on appelle le «produit minimum viable» : ça peut être un prototype papier, ça peut être un formulaire Google, dont l'objectif est de valider l'hypothèse de base avant même de continuer. Par exemple au lieu de faire un site web de location de robes de gala par Internet, on va faire un prototype papier, on va sortir dans la rue et on va faire un sondage - sans avoir besoin de créer le site - afin de valider certaines hypothèses de départ et le cas échéant faire le site. Je pense que cette approche est intéressante pour tout ce qui est développement de produits, notamment de produits de communication. Ici, la recherche, mais ce n'est pas au sens de la recherche universitaire, est importante. Elle se fait sur un modèle trial and error : on cherche, on teste, on mesure, on revient. C'est plus de la recherche au sens scientifique, comme un biologiste qui essaie d'évaluer l'interaction de deux produits. On n'est presque plus dans les sciences sociales, ou en tout cas on utilise alors un système scientifique d'observation.

Ce n'est pas facile d'introduire une telle méthodologie en organisation. Ça demande que le management fasse complètement confiance, car il peut s'agir de 
246 Le communicateur bousculé par le numérique

tester cinq hypothèses en parallèle - donc avec un fort investissement au départ pour un résultat qui peut être au final décevant: il est possible de n'arriver finalement à faire sortir aucun produit, de ne rien avoir. C'est davantage du domaine de la recherche, car le résultat peut-être complètement différent ou très éloigné de ce qu'on imaginait au départ. Ça demande un changement de manière de penser puisqu'il faut poser au départ qu'on ne sait pas, qu'on a des idées mais qui doivent être testées.

C'est une posture, une manière de voir différente, il faut tester, essayer, expérimenter... Par ailleurs, les praticiens de la communication numérique doivent essayer d'utiliser les outils pas seulement dans le contexte professionnel. Dans mon cas, j'ai des blogs personnels, j'utilise les réseaux sociaux personnellement. Quand j'écris dans un blog, je vois si les tags que je mets fonctionnent, l'article qui a le plus de plus visiteurs, etc. Autrement dit, je teste. En fait c'est un métier d'expérimentateur. Il faut apprendre à apprendre. Peut-être le rôle de l'université dans le champ des sciences de l'information et de la communication est d'abord d'apprendre à apprendre et apprendre à évoluer - afin notamment de pouvoir s'adapter continuellement à l'environnement qui évolue. 